\title{
ORIGINAL ARTICLE \\ Investigating the status of using lower extremity orthoses recommended to patients with spinal cord injury
}

\author{
E Koyuncu, GF Nakipoğlu Yüzer, P Çam and N Özgirgin
}

\begin{abstract}
Study design: Retrospective descriptive study.
Objectives: The present study aimed to investigate the rate of using orthosis among spinal cord injury (SCI) patients for whom orthosis was recommended for standing and walking, the relationship between the clinical and demographic characteristics of SCl and the use of orthosis and the reasons for not using orthosis.

Setting: Ankara Physical Medicine and Rehabilitation Training and Research Hospital, Turkey.

Methods: The study included $62 \mathrm{SCl}$ patients for whom orthosis was recommended for standing and ambulation. The patients were classified into two groups as individuals using and not using the recommended orthosis every day in order to evaluate the effect of age, gender, residence, duration of disease/recommended duration of orthosis, recommended orthosis, lesion level-degree, lower extremity tonus-range of motion and ambulation level on the frequency of orthosis use.

Results: The orthosis most commonly recommended was hip-knee-ankle-foot orthosis with waist or pelvic belt (45.2\%). Of the patients, $25.8 \%$ have never used the orthosis. The most common reason for not using the recommended orthosis was the failure to facilitate the daily life activities of the patient (30\%), the difficulties in putting them on and taking them off (20\%), the belief that it is unnecessary (15\%) and the pressure (15\%). In addition, the assessed clinical and demographic features were detected as not important risk factors for not using orthosis.
\end{abstract}

Conclusion: At least one out of four patients with $\mathrm{SCl}$ do not use the recommended lower extremity orthosis. Selecting eligible patients, patient training and follow-up are important for increased frequency of orthosis usage.

Spinal Cord (2016) 54, 996-1000; doi:10.1038/sc.2016.39; published online 15 March 2016

\section{INTRODUCTION}

In patients with spinal cord injury (SCI) characterized by loss of movement and sense, lower the lesion level, the degree of loss of ability to stand and walk determines the functional status. ${ }^{1}$ Enabling independent mobilization of the patient after the acute period is one of the important goals in the rehabilitation of SCI patients. Various orthoses are used for this purpose. ${ }^{2}$ Many SCI patients can provide their transfer with a similar speed and energy consumption to normal individuals by using wheelchair. However, using wheelchair is associated with some problems such as limited transfer with wheelchair because of environmental factors, pressure sore related to longterm sitting, osteoporosis and joint deformity risk. ${ }^{1}$ In addition, standing and walking are known to have some advantages such as reducing osteoporosis, preventing pressure sore, improving bladder and bowel functions, circulatory and digestive system and sleeping, enhancing general well-being and reducing pain and fatigue in SCI patients in addition to providing transfer and increasing independency. ${ }^{1,3-5}$ Therefore, several different orthotic devices such as mechanical orthoses, functional electrical systems, and hybrid and robotic systems have been developed for SCI patients for standing and walking purposes. ${ }^{6-9}$ However, using orthosis has some disadvantages such as high-energy consumption during ambulation, ${ }^{10-12}$ slow walking speed, ${ }^{12}$ not feeling safe on them, ${ }^{13}$ and the difficulties experienced and the time spent while putting them on and taking them off without assistance. ${ }^{14}$ Such disadvantages limit the use of orthosis. Previous studies reported that $27-58.2 \%{ }^{13-17}$ of various orthoses recommended for standing or walking are not used. This leads to the loss of the benefits from orthosis use, as well as the waste of resources. On the basis of the literature review, some studied have investigated the reasons for not using orthosis in SCI patients; however, there are limited up-to-date studies, ${ }^{16}$ and there is very limited research examining the relationship between the rate of using orthosis and clinical and demographic characteristics. ${ }^{13,16}$

It is important to determine the reasons for terminating the use of orthosis and the relationship between the rate of using orthosis and clinical and demographic characteristics of SCI patients for taking necessary precautions to increase the frequency of using orthosis. Therefore, the present study aimed to investigate the rate of using orthosis among SCI patients for whom orthosis was recommended for standing and walking, the relationship between the clinical and demographic characteristics of SCI and the use of orthosis and the reasons for not using orthosis.

\section{MATERIALS AND METHODS}

This retrospective descriptive study was approved by the Training Planning and Coordination Committee of our hospital. 
The study included 62 SCI patients who were discharged from our hospital between 2012 and 2014, for whom orthosis was recommended for standing and ambulation and who could be accessed. Individuals with additional neurological diseases were excluded.

Clinical and demographic characteristics of the patients were evaluated through the Hospital Information Management System (HIMS). Patients' age, gender, duration of the disease, SCI etiology, functional levels, SCI level and degree according to the American Spinal Cord Injury Association (ASIA) Impairment Scale (AIS), lower and upper extremity range of motion (ROM) and tonus were recorded. The participants were deeply asked all the necessary questions regarding the use of their orthoses. They were interviewed about the frequency of using orthosis and the reasons for not using. Recommended orthosis, recommended duration of orthosis, frequency of using orthosis and reasons for not using were questioned. The importance of orthosis use was told in detail to the all patients. We also recommended doctor controls if there is a problem they faced during the orthosis usage.

Verticalization or ambulation in parallel bar was defined as therapeutic ambulation, and mobilization using an orthosis to implement daily life activities inside or outside of the house was defined as functional ambulation. The patients were classified into two groups based on the duration of disease/ recommended duration of orthosis as $0-12$ months and more than 12 months. The residential locations (city, district or village) of the patients were determined in order to evaluate the effect of residence on using orthoses. The patients were classified into two groups as individuals using and not using

\section{Table 1 Demographic and clinical characteristics of the patients} $(n=62)$

\begin{tabular}{lc}
\hline Age, years (mean \pm s.d.), median & $35.7 \pm 14.7,33.5(15-81)$ \\
(min-max) & \\
$\begin{array}{l}\text { Gender }(\mathrm{n}, \%) \\
\quad \text { Female/male }\end{array}$ & $16(25.8 \%) / 46(74.2 \%)$ \\
Residence (n, \%) & \\
$\quad$ City/district-village & $28(45.2 \%) / 34(54.8 \%)$
\end{tabular}

Disease duration (days)

Mean \pm s.d./median (min-max)

$1476 \pm 2074 / 825(150-11520)$

Disease duration (n, \%)

0-12 months/> 12 months

$15(24.2 \%) / 47(75.8 \%)$

Level of lesion (n, \%)

Cervical/Thoracic/Lumbar

$7(11.3 \%) / 37(59.7 \%) / 18(29 \%)$

Degree of injury ( $\mathrm{n}, \%)$

AIS A+B/AIS C+D

$38(61.3 \%) / 24(38.7 \%)$

Lower extremity range of motion (n, \%)

Not limited/limited

$45(72.6 \%) / 17(27.4 \%)$

Lower extremity tonus

Spasticity yes/no

$24(38.7 \%) / 38(61.3 \%)$

Ambulation status (n, \%)

Therapeutic/functional

ambulation

$53(85.5 \%) / 9(14.5 \%)$

Etiology (n, \%)

Falling from a height $\quad 29(46.8 \%)$

Motor vehicle accident $20(32.3 \%)$

Non-traumatic $7(11.3 \%)$

Gunshot wound $2(3.2 \%)$

Crush injury

$4(6.5 \%)$ the recommended orthosis every day in order to evaluate the effect of age, gender, residence, duration of disease/recommended duration of orthosis, recommended orthosis, lesion level-degree, lower extremity tonus-ROM and ambulation level on the frequency of orthosis use.

\section{Statistical analysis}

Statistical analyzes were performed by using SPSS version 11.5 software (SPSS, Inc, 2002, Chicago, IL, USA). The Kalmogorov-Smirnov test was used to determine whether the continuous variable distribution was normal. Descriptive statistics were provided as mean+standard deviation or median (minimum-maximum) for continuous variables, whereas the number of cases and percentages were used for nominal variables. The Mann-Whitney's $U$-test was used to determine whether a statistically significant difference was present for orderable variables not normally distributed. The $\chi^{2}$ and the Fisher exact tests were used for categorical comparisons. Binary logistic regression analysis was performed in patients with SCI after investigating the relation of orthosis use frequency with clinical and demographic features by using univariate analysis. A $P$-value of $<0.05$ was considered statistically significant.

\section{RESULTS}

Table 1 presents the demographic and clinical characteristics of 62 SCI patients, $74.2 \%$ of whom were male and the mean age of whom was $35.7 \pm 14.7$ years. The orthosis most commonly recommended was Hip-Knee-Ankle-Foot orthosis (HKAFO) with waist or pelvic belt (45.2\%). Of the patients, $67.7 \%$ were using the recommended orthosis every day, whereas $25.8 \%$ have never used the orthosis. In addition, $6.5 \%$ of the patients were not using the recommended orthosis every day but using at least once a week. The most common reason for not using the recommended orthosis was the failure to facilitate the daily life activities of the patient (30\%; Table 2).

Age, gender, residence, the disease duration and recommended duration of orthosis, recommended orthosis, lesion level, ROM limitation in lower extremities, presence of spasticity and ambulation level were not statistically significantly related to the frequency of orthosis use $(P>0.05)$. The rate of motor complete patients $(71.4 \%)$ was statistically significantly higher in the group using the

Table 2 Demographic and clinical characteristics of the patients $(n=62)$

\begin{tabular}{lc}
\hline Orthosis (n, \%) \\
pAFO/mAFO/pKAFO/mKAFO/mHKAFO & $7(11.3 \%) / 5(8.1 \%) / 13(21 \%) / 9$ \\
with waist or pelvic belt & $(14.5 \%) / 28(45.2 \%)$
\end{tabular}

Time since prescription of the orthosis (days)

Mean + s.d./median (min-max)

$663+632 / 485(60-3600)$

Time since prescription of the orthosis (n, \%)

0-12 months/>12 months

$26(41.9 \%) / 36(58.1 \%)$

Frequency of orthosis use (n, \%)

Every day/1-7 times a week/none

$42(67.7 \%) / 4(6.5 \%) / 16(25.8 \%)$

Non-use reasons of patients who do not use the recommended

orthosis every day (n, \%)

Failure to facilitate daily life $\quad 6(30 \%)$

Difficulty to wear $4(20 \%)$

Belief that it is unnecessary $3(15 \%)$

Cause pressure $3(15 \%)$

Environmental conditions $\quad 2(10 \%)$

Presence of pressure sore $1(5 \%)$

Device aging $1(5 \%)$

Abbreviations: mAFO, metal Ankle-Foot orthosis; mHKAFO, metal Hip-Knee-Ankle-Foot orthosis; mKAFO, metal Knee-Ankle-Foot orthosis; pAFO, plastic Ankle-Foot Orthosis; pKAFO, plastic Knee-Ankle-Foot orthosis. 
Table 3 The relationship between the clinical and demographic characteristics of patients and the use of orthosis

\begin{tabular}{|c|c|c|c|}
\hline & $\begin{array}{l}\text { Patients using recommended } \\
\text { orthosis every day }(n=42)\end{array}$ & $\begin{array}{c}\text { Patients not using recommended } \\
\text { orthosis every day }(n=20)\end{array}$ & P-value \\
\hline Age, years (mean \pm s.d.), median (min-max) & $33.5 \pm 12.1,31(18-60)$ & $40.4 \pm 18.6,42(15-81)$ & $0.185^{a}$ \\
\hline Female/male & $10(23.8 \%) / 32(76.2 \%)$ & $6(30 \%) / 14(70 \%)$ & \\
\hline Residence (n, \%) & & & $0.573^{b}$ \\
\hline City/district-village & $20(47.6 \%) / 22(52.4 \%)$ & $8(40 \%) / 12(60 \%)$ & \\
\hline Time since prescription of the orthosis $(n, \%)$ & & & $0.445^{b}$ \\
\hline $0-12$ ay/ $>12$ ay & $19(45.2 \%) / 23(54.8 \%)$ & $7(35 \%) / 13(65 \%)$ & \\
\hline Level of lesion (n, \%) & & & $0.671^{c}$ \\
\hline Cervical/thoracolumbar & $4(9.5 \%) / 38(90.5 \%)$ & $3(15 \%) / 17(85 \%)$ & \\
\hline Degree of injury $(n, \%)$ & & & $0.018^{b}$ \\
\hline AIS A+B/AIS C+D & $30(71.4 \%) / 12(28.6 \%)$ & $8(40 \%) / 12(60 \%)$ & \\
\hline Ambulation status (n, \%) & & & $0.133^{c}$ \\
\hline Therapeutic/functional ambulation & $38(90.5 \%) / 4(9.5 \%)$ & $15(75 \%) / 5(25 \%)$ & \\
\hline Orthosis $(n, \%)$ & & & $0.018^{b}$ \\
\hline Ankle-foot orthosis & $4(33.3 \%)$ & $8(66.7 \%)$ & \\
\hline Knee-Ankle-Foot orthosis & $17(77.3 \%)$ & $5(22.7 \%)$ & \\
\hline mHKAFO with waist or pelvic belt & $21(75 \%)$ & $7(25 \%)$ & \\
\hline
\end{tabular}

Abbreviations: AIS, American Spinal Cord Injury Association (ASIA) Impairment Scale; mHKAFO, metal Hip-Knee-Ankle-Foot orthosis.

The recommended orthosis and the status of using orthosis were compared using the $\chi^{2}$-test, and paired comparisons were made as $P$-value was found $<0.05$ ( $P=0.018$ ), and evaluated using

Bonferroni's correction. The results from paired comparisons revealed that all three $P$-values were $>0.017$, and therefore no statistically significant correlation was found between the recommended orthosis and the status of using orthosis.

a Mann-Whitney's U-test.

b $\chi^{2}$-test.

'Fisher's Exact test.

recommended orthosis every day compared with the group not using the orthosis every day $(40 \% ; P=0.018$; Table 3$)$. As a result of binary logistic regression analysis, the whole clinical and demographic variables included in the model were found unimportant factors for the frequency of orthosis usage $(P>0.05$; Table 4$)$.

Table 5 presents the clinical characteristics and the reasons for not using the orthosis of 20 patients who were not using the recommended orthosis every day.

\section{DISCUSSION}

The most commonly recommended orthosis for the study participants composed of SCI patients most of whom (71\%) had cervical or thoracic level injuries was HKAFO with waist or pelvic belt. HKAFO with waist or pelvic belt was recommended to the patients who were unable to have functional-community mobilization with wheelchair because of socio-economic and environmental reasons for therapeutic purposes. The present study found that $67.7 \%$ of the SCI patients were using the recommended orthosis every day, whereas $25.8 \%$ have never used the orthosis. Previous studies reported that $27-58.2 \%{ }^{13-17}$ of various orthoses recommended for standing or ambulation are not used. The different rates may be due to the differences in the orthoses investigated for the usage rate and other methodological differences. For instance, the study by Sykes et al. ${ }^{15}$ investigated only the frequency of using the reciprocating gait orthosis and found the non-use rate as $38 \%$. The study by Coghlan et al. ${ }^{17}$, was conducted with 98 paraplegic patients recommended using long leg orthosis and found that $58.2 \%$ have never used the recommended orthosis. The higher non-use rate of orthosis compared with the present study can be resulted from the patients' longer mean duration of the disease ( 6.5 years) and the inclusion of the only long leg orthosis-recommended to the paraplegic patients. ${ }^{17}$ A study that was conducted with paraplegic SCI patients recommending Ankle-Foot orthosis, Knee-Ankle-Foot orthosis and HKAFO with waist or pelvic belt found the non-use rate of orthoses as $27 \%$. Another study with 35 patients found the orthosis usage rate as $31 \%$, a close rate to that found in the present study. In that study, $\mathrm{KAFO}$ was recommended to $54.3 \%$ and $\mathrm{AFO}$ was recommended to $45.7 \%$ of the patients. Although studies report different non-use rates of lower extremity orthoses in SCI patients, it is ultimately seen that at least one out of four patients do not use the orthosis. This leads to the loss of the benefits from orthosis use, as well as the waste of resources. Therefore, it is important to determine why SCI patients terminate using orthosis and which clinical/demographic characteristics affect orthosis usage in order to increase the orthosis usage rates.

It has been determined in a study by Mikelberg et al. ${ }^{14}$ that the most common reason for not using orthosis in SCI patients is the fact that it is too difficult and time consuming to use an orthosis. The other reasons stated were the lack of suitable space in the home environment for proper use, pressure sore, weight change, poor adjustment, winter conditions and fractures. ${ }^{14}$ A study conducted by Hong et al. ${ }^{13}$ with 73 SCI patients determined the reasons for not using orthosis as the extremely slow, hard and insecure use of orthosis (35.1\%), the impracticality (29.8\%), being too weak (21.1\%), broken leg/hip (3.4\%), leg amputations (5.3\%) and muscle spasm (5.3\%). The study by Alemdaroğlu et al. ${ }^{16}$ established the reasons as the no longer presence of the need due to improvement, the tightening caused by the orthosis, the discomfort, the need for assistance to wear, its weight, 
Table 4 Binary logistic analysis of clinical and demographic characteristics that can be related to the use of orthosis

\begin{tabular}{|c|c|c|c|}
\hline & Odds ratio & $95 \% \mathrm{Cl}$ & P-value \\
\hline Age & 1.047 & $0.989-1.108$ & 0.116 \\
\hline \multicolumn{4}{|l|}{ Gender } \\
\hline Female (baseline) & 0.881 & $0.170-4.562$ & 0.880 \\
\hline \multicolumn{4}{|l|}{ Male } \\
\hline \multicolumn{4}{|l|}{ Residence } \\
\hline City (baseline) & 2.201 & $0.526-9.205$ & 0.280 \\
\hline \multicolumn{4}{|l|}{ District-village } \\
\hline \multicolumn{4}{|l|}{ Disease duration } \\
\hline 0-12 months (baseline) & 1.903 & $0.320-11.330$ & 0.480 \\
\hline \multicolumn{4}{|l|}{$>12$ months } \\
\hline \multicolumn{4}{|l|}{ Level of lesion } \\
\hline Cervical & 3.499 & $0.380-32.247$ & 0.269 \\
\hline \multicolumn{4}{|l|}{ Thoracolumbar (baseline) } \\
\hline \multicolumn{4}{|l|}{ Degree of injury } \\
\hline AIS A+B (baseline) & 3.998 & $0.722-22.139$ & 0.112 \\
\hline \multicolumn{4}{|l|}{ AIS C+D } \\
\hline \multicolumn{4}{|l|}{ Lower extremity range of motion } \\
\hline Not limited (baseline) & 5.297 & 0.957-29.302 & 0.056 \\
\hline \multicolumn{4}{|l|}{ Limited } \\
\hline \multicolumn{4}{|l|}{ Lower extremity tonus } \\
\hline Spasticity yes & 0.148 & $0.022-1.003$ & 0.052 \\
\hline \multicolumn{4}{|l|}{ Spasticity no (baseline) } \\
\hline \multicolumn{4}{|l|}{ Ambulation status } \\
\hline Therapeutic & 0.989 & $0.099-9.847$ & 0.992 \\
\hline \multicolumn{4}{|l|}{ Functional ambulation (baseline) } \\
\hline \multicolumn{4}{|l|}{ Orthosis } \\
\hline \multicolumn{4}{|l|}{ Ankle-Foot orthosis (baseline) } \\
\hline Knee-Ankle-Foot orthosis & 0.145 & $0.014-1.446$ & 0.100 \\
\hline mHKAFO with waist or pelvic belt & 0.295 & $0.025-3.543$ & 0.336 \\
\hline
\end{tabular}

Abbreviations: AIS, American Spinal Cord Injury Association (ASIA) Impairment Scale; $\mathrm{Cl}$, confidence interval; mHKAFO, metal Hip-Knee-Ankle-Foot orthosis.

the tiredness caused by its use, the unsuitability to use outside the house, development of fractures and spasticity. A study with 98 paraplegic patients found the reasons for not using orthosis as the easiness of transfer with wheelchair, the extended time and the difficulties in putting them on and taking them off, impracticality, the higher amount of energy required to walk with an orthosis, unreliability, pressure sores, increased pain and spasticity, being not a close-fit, hip and leg fractures, unsuitability of environmental media to orthosis usage and shoulder pain. ${ }^{14}$ As a result, the recommended orthoses cannot be used most commonly because of reasons such as failure to facilitate daily life, the difficulties in putting them on and taking them off, the belief that it is unnecessary, inappropriate design and wearing out of orthosis, pressure applied by the orthosis due to the factors such as changes in body weight and environmental conditions. Given the results of the study, it is believed that, first, the choice of the suitable orthosis to the suitable patient by the rehabilitation team also including an orthotist is important. During the orthosis decision, attention should be paid to get the patient opinions about the orthosis usage, to inform and train the patient about the orthosis. Training should specifically involve how to use an orthosis safely, and it should be emphasized that mobilization is not the sole purpose of using an orthosis and orthoses have positive effects also on other systems. It is believed that the factors that would increase the rates of using orthosis include making the environmental media suitable to use an orthosis, manufacturing an easier, lighter and a close-fit orthoses according to the patient's body size, and periodical follow-ups of the patient for changes in the orthosis in cases such as orthosis aging/weight changes/patient improvement or worsening.

The present study concluded that the frequency of using the recommended orthosis was not affected by age, gender, residence, the disease and recommended duration of orthosis, recommended orthosis, lesion level, ROM limitation in lower extremities, presence of spasticity and ambulation level. A study conducted with paraplegic patients established no significant difference in age, the disease and recommended duration of orthosis, length of hospital stay, and the lesion level (between T10 and above, and T10 and below) between patients who were using and not using the recommended orthosis. The rate of terminating orthosis usage was found significantly higher in incomplete SCI patients (82\%) compared with complete patients $(18 \%)$. In that study, $36 \%$ of the patients terminating the orthosis usage stated the reason of termination as improvement. The study reported that the higher improvement rate in patients with incomplete disease and lower lesion levels might lead to increased termination of orthosis usage. ${ }^{16}$ Although the present study did not find any difference in orthosis usage among cervical and thoracolumbar SCI patients, it demonstrates that the rates of not using orthosis are higher in motor incomplete patients. However, the study by Hong et al. ${ }^{13}$ established a higher non-use frequency for orthosis in SCI patients with a higher level and a higher lesion degree. It was reported that the higher amount of energy required for ambulation in high-level SCI patients might result in less use of orthosis in such patients. Besides, it was found that the frequency of not using orthosis was higher in SCI patients who were older, had comorbid medical conditions and had higher levels of dependence in daily life activities. ${ }^{13}$ When the results of the present and other studies were evaluated, it was determined that SCI patients with incomplete or lower-level lesions seem to give up orthosis usage because of the higher rate of improvement-namely, reduced need to the orthosis use. However, it is assumed that the SCI patients with higher level and high degree lesion in addition to the higher rate of dependency in their daily life activities can give up the use of orthosis because of reasons such as the necessity of more energy required for ambulation and more difficulties in orthosis usage. In conclusion, the most important factors determining the rate of orthosis usage are the level of lesion, degree of injury and dependency in daily living activities.

\section{Limitation}

The retrospective design can be considered as the main limitation of the present study. Prospective studies that would be conducted with a larger patient population will reveal the relationship between both the frequency of orthosis usage and the reasons for not using orthosis, and the clinical and demographic characteristics in a clearer way.

\section{CONCLUSION}

At least one out of four patients with SCI do not use lower extremity orthoses recommended. Age, gender, residence, the disease duration and recommended duration of orthosis, recommended orthosis, level 
Table 5 Clinical characteristics and non-use reasons of patients who do not use the orthosis every day $(n=20)$

Non-use reasons of patients who do not use

the recommended orthosis every day

Orthosis

Level and degree of injury

Functional status

Environmental conditions

Environmental conditions

Presence of pressure sore

Device aging

Belief that it is unnecessary

Belief that it is unnecessary

Belief that it is unnecessary

Difficulty to wear

Difficulty to wear

Difficulty to wear

Difficulty to wear

Failure to facilitate daily life

Failure to facilitate daily life

Failure to facilitate daily life

Failure to facilitate daily life

Failure to facilitate daily life

Failure to facilitate daily life

Cause pressure

Cause pressure

Cause pressure

$\begin{array}{ll}\text { mHKAFO with waist belt } & \text { T10 AIS A } \\ \text { mKAFO } & \text { L1 AIS C } \\ \text { mKAFO } & \text { L1 AIS B } \\ \text { mHKAFO with pelvic belt } & \text { T10 AIS A } \\ \text { pAFO } & \text { L2 AIS D } \\ \text { mHKAFO with waist belt } & \text { L1 AIS C } \\ \text { pAFO } & \text { L1 AIS C } \\ \text { mHKAFO with waist belt } & \text { T7 AIS A } \\ \text { mAFO } & \text { L3 AIS D } \\ \text { pAFO } & \text { L2 AIS C } \\ \text { mKAFO } & \text { C4 AIS D } \\ \text { mAFO } & \text { T12 AIS D } \\ \text { mHKAFO with waist belt } & \text { T12 AIS A } \\ \text { mAFO } & \text { L2 AIS C } \\ \text { mHKAFO with waist belt } & \text { T12 AIS A } \\ \text { pKAFO } & \text { L2 AIS D } \\ \text { pKAFO } & \text { C4 AIS D } \\ \text { mAFO } & \text { L2 AIS B } \\ \text { mHKAFO with waist belt } & \text { T7 AIS A } \\ \text { pAFO } & \text { C6 AIS D }\end{array}$

Therapeutic ambulation Therapeutic ambulation Therapeutic ambulation Therapeutic ambulation Therapeutic ambulation Therapeutic ambulation Functional ambulation Therapeutic ambulation Functional ambulation Therapeutic ambulation Therapeutic ambulation Functional ambulation Therapeutic ambulation Therapeutic ambulation Therapeutic ambulation Therapeutic ambulation Therapeutic ambulation Functional ambulation Therapeutic ambulation Functional ambulation

Abbreviations: AIS, American Spinal Cord Injury Association (ASIA) Impairment Scale; C, cervical; L, lumbar; mAFO, metal Ankle-Foot orthosis; mHKAFO, metal Hip-Knee-Ankle-Foot orthosis; mKAFO, metal Knee-Ankle-Foot orthosis; pAFO, plastic Ankle-Foot orthosis; pKAFO, plastic Knee-Ankle-Foot orthosis; T, thoracic.

and degree of lesion, ROM limitation in lower extremities, presence of spasticity and ambulation level were not statistically significantly related to the frequency of orthosis use. The recommended orthosis cannot be used most commonly because of the reasons such as failure to facilitate daily life, the difficulties in putting them on and taking them off, the belief that it is unnecessary, inappropriate design and wearing out of orthosis, pressure applied by the orthosis due to the factors like changes in body weight and environmental conditions. Given the results of the study, it is believed that, first, the choice of the suitable orthosis to the suitable patient by the rehabilitation team also including an orthotist is important. Follow-ups of the patient for changes in the orthosis in cases such as orthosis aging/weight changes/ patient improvement or worsening are important for increased frequency of orthosis usage.

\section{DATA ARCHIVING}

There were no data to deposit.

\section{CONFLICT OF INTEREST}

The authors declare no conflict of interest.

1 Karimi MT. Evidence-based evaluation of physiological effects of standing and walking in individuals with spinal cord injury. Iran J Med Sci 2011; 36: 242-253.

2 Nas K, Yazmalar L, Şah V, Aydın A, Öneş K. Rehabilitation of spinal cord injuries. World J Orthop 2015; 6: 8-16.
3 Eng JJ, Levins SM, Townson AF, Mah-Jones D, Bremner J, Huston G. Use of prolonged standing for individuals with spinal cord injuries. Phys Ther 2001; 81: 1392-1399.

4 Biering-Sørensen F, Hansen B, Lee BS. Non-pharmacological treatment and prevention of bone loss after spinal cord injury: a systematic review. Spinal Cord 2009; 47: 508-518.

5 Correa GI, Fuentes M, Gonzalez X, Cumsille F, Piñeros JL, Finkelstein J. Predictive factors for pressure ulcers in the ambulatory stage of spinal cord injury patients. Spinal Cord 2006; 44: 734-739.

6 Karimi MT. What are the next steps in designing an orthosis for paraplegic subjects? Int J Prev Med 2012; 3: 145-159.

7 Kobetic R, To CS, Schnellenberger JR, Audu ML, Bulea TC, Gaudio R et al. Development of hybrid orthosis for standing, walking, and stair climbing after spinal cord injury. J Rehabil Res Dev 2009; 46: 447-462.

8 Louie DR, Eng JJ, Lam T. Spinal Cord Injury Research Evidence (SCIRE) Research Team. Gait speed using powered robotic exoskeletons after spinal cord injury: a systematic review and correlational study. J Neuroeng Rehabil 2015; 12: 82.

9 Hardin EC, Kobetic R, Triolo RJ. Ambulation and spinal cord injury. Phys Med Rehabil Clin N Am 2013; 24: 355-370.

10 Cerny D, Waters R, Hislop H, Perry J. Walking and wheelchair energetics in persons with paraplegia. Phys Ther 1980; 60: 1133-1139.

11 Merati G, Sarchi P, Ferrarin M, Pedotti A, Veicsteinas A. Paraplegic adaptation to assisted-walking: energy expenditure during wheelchair versus orthosis use. Spinal Cord 2000; 38: 37-44.

12 Waters RL, Lunsford BR. Energy cost of paraplegic locomotion. J Bone Joint Surg Am 1985; 67: 1245-1250.

13 Hong C, San Luis EB, Chung S. Follow-up study on the use of leg braces issued to spinal cord injury patients. Paraplegia 1990; 28: 172-177.

14 Mikelberg R, Reid S. Spinal cord lesions and lower extremity bracing: an overview and follow-up study. Paraplegia 1981; 19: 379-385.

15 Sykes L, Edwards J, Powell ES, Ross ER. The reciprocating gait orthosis: long-term usage patterns. Arch Phys Med Rehabil 1995; 76: 779-783.

16 Alemdaroğlu E, Mandıroğlu S, Uçan H, Çelik C. The continuity of orthosis use by paraplegics which had been prescribed during in-patient rehabilitation. Turk J Phys Med Rehab 2014; 60: 223-230.

17 Coghlan JK, Robinson CE, Newmarch B, Jackson G. Lower extremity bracing in paraplegia—a follow-up study. Paraplegia 1980; 18: 25-32. 